\title{
On-orbit Characterization of the MODIS SDSM Screen for Solar Diffuser Degradation Estimation
}

\author{
Hongda Chen $^{\mathrm{a}}$, Xiaoxiong Xiong ${ }^{\mathrm{b}}$, Amit Angal ${ }^{\mathrm{a}}$ and Kevin A. Twedt ${ }^{\mathrm{a}}$ \\ ${ }^{\text {a} S c i e n c e ~ S y s t e m s ~ a n d ~ A p p l i c a t i o n s ~ I n c ., ~} 10210$ Greenbelt Road, Lanham, MD 20706 \\ ${ }^{\mathrm{b}}$ Sciences and Exploration Directorate, NASA Goddard Space Flight Center, Greenbelt, MD, 20706
}

Abstract - MODIS reflective solar bands (RSB) are calibrated on-orbit using a solar diffuser (SD) with its degradation tracked by an on-board solar diffuser stability monitor (SDSM). The SDSM has nine detectors with wavelengths from 0.41 to $0.94 \mu \mathrm{m}$. It is operated during each scheduled SD calibration event, making alternate observations of the Sun and the SD. Due to erroneous design parameters, which led to misalignment of the key elements in the SDSM, there are significant ripples in the Sun view responses as the solar viewing angle changes. At the mission beginning, the effect of the ripples was eliminated by normalizing each SDSM detector response to the response of detector 9 (D9) at 0.94 $\mu \mathrm{m}$, assuming that $\mathrm{D} 9$ had no degradation. However, D9 degradation increases over MODIS operation times. Degradation of up to $2 \%$ has been recently observed in $\mathrm{D} 9$ for Terra MODIS. A newly implemented approach reduces the Sun view ripples using a look-up table (LUT) constructed using SDSM data carefully selected from a short period early in the mission lifetime. In this paper, we provide an overview of different approaches that have been applied over the years by the MODIS Characterization Support Team (MCST) to track the on-orbit SD degradation. We evaluate the overall SD and SDSM onorbit performance for both Terra and Aqua MODIS, as well as the impact on the MODIS RSB calibration uncertainty.
Index Terms - MODIS, solar diffuser, solar diffuser stability monitor, calibration, radiometer

\section{INTRODUCTION}

MODIS is currently operated onboard the Earth Observing System (EOS) Terra and Aqua spacecraft launched on December 18, 1999 and May 4, 2002, respectively [1-3]. It is a cross-track scanning radiometer with 36 spectral bands. Bands 1-19 and 26 with wavelengths from 0.41 to $2.2 \mu \mathrm{m}$ are the reflective solar bands (RSB), and bands 2025 and 27-36 with wavelengths from 3.7 to $14.5 \mu \mathrm{m}$ are the thermal emissive bands (TEB). In order to monitor and maintain the quality of the calibrated data products, MODIS was designed with a set of on-board calibration (OBC) subsystems, including a solar diffuser (SD), a solar diffuser stability monitor (SDSM), a spectroradiometric calibration assembly (SRCA), and a vgrooved Blackbody (BB). The SRCA is primarily used for the sensor's spectral (RSB only) and spatial (TEB and RSB) characterization. The SD and SDSM are used together for RSB calibration and the $\mathrm{BB}$ is used for TEB calibration. In addition, there is a space view port providing a view of cold space to allow for a zero radiance reference measurement for each scan. 
The RSB are calibrated using the SD, which has a bi-directional reflectance factor (BRF) that was characterized pre-launch. On-orbit variations of the SD BRF are tracked by the SDSM, which has nine filtered detectors embedded in a solar integration sphere (SIS) to collect data over wavelengths ranging from 0.41 to $0.94 \mu \mathrm{m}$. Table 1 shows the center wavelengths of the SDSM detectors, which are closely matched to some of the MODIS spectral bands. The SD degradation derived from the SDSM can be interpolated to apply to the MODIS RSB. Normally the SDSM is operated during each SD calibration, making alternate views of the Sun, the SD, and the dark background via a rotating mirror.

The SDSM was designed as a ratioing radiometer: it tracks the SD BRF degradation by providing regular measurements of the ratio of the background corrected SD response to the background corrected Sun view response for each SDSM detector [4-8]. The SDSM is only able to track the on-orbit BRF changes at a fixed angle. The BRF is view angle dependent and the angular dependence was well characterized prelaunch. Since launch, two sets of spacecraft yaw maneuvers, covering the solar illumination angles, have been performed for Terra MODIS and one for Aqua MODIS to monitor the SD BRF uniformity. However these were only performed in early mission, and they cannot be used to monitor any timedependent change in the angular dependence. Thus, the computed SD degradation as measured by the SDSM is applied as a multiplicative scalar.

An attenuation screen consisting of a twodimensional array of pinholes is present in the Sun view path to prevent detector saturation when viewing the Sun. However, due to a design error, which led to misalignment of the screen relative to other optical elements in the SDSM sub-system, significant ripples are observed in the SDSM Sun view responses $[4,9]$. The throughput of the screen is not expected to depend on solar viewing angle, but the amount of light that passes through the SIS entrance aperture changes with angle due to this misalignment. The effect has been modeled before and the general shape of the sun view ripples during a single calibration was predicted reasonably well [4]. Ripples on the order of $\pm 5 \%$ are observed during a single calibration (a fraction of one orbit) as the solar angle changes from scan to scan. Similar size ripples are also observed when monitoring the Sun view response as the solar angle changes over the course of a yearly cycle. This made it very difficult to accurately track the SD degradation using the originally designed ratioing approach, especially early in the mission when there was not enough data to fully characterize the yearly oscillations.

In order to improve the calibration, the MODIS Characterization Support Team (MCST) at NASA/GSFC developed an alternative calibration approach [5] that involved normalizing the response of each detector to that of detector 9 (D9). This approach relies on the fact that the pattern of the ripples is consistent across the SDSM detectors and the SD BRF degradation at the D9 wavelength $(0.94 \mu \mathrm{m})$ is extremely small $[10,11]$. After normalization to D9, the ripples in the response of the other eight SDSM detectors are effectively removed. The normalization approach produces very reliable trends in the SD degradation at all SDSM wavelengths except for D9. For a short operational period, the SD degradation of D9 can be assumed to be negligible. But as both MODIS instruments are operating well beyond their design life, the accumulated 
degradation at D9 wavelength must be analyzed and corrected.

To mitigate this deficiency, we have developed two geometrical compensation approaches that can effectively reduce the impact of the screen mis-alignment and the corresponding Sun view ripples for all SDSM detectors. The first approach uses a look-up table (LUT) constructed from SDSM D9 observations in a short period over a range of viewing angles. The LUT is used to directly determine the D9 degradation, and the degradation of the remaining eight detectors is determined by combining this with the previous normalization strategy. This is the approach currently implemented for RSB calibration in the MODIS Collection 6 Level 1B (C6 L1B) products. More recently, we have also developed a dynamic scheme to extend the LUT-based approach to other detectors, so as to provide even more precise results.

In this paper, we provide an overview of various approaches developed to track MODIS SD on-orbit degradation, with more emphasis on the LUT-based approaches. In Section II, we describe the three main methodologies that have been used. In Sections III and IV, we focus on the details of the LUT-based methods and demonstrate their advantages, and in Section V we show the results of SDSM and SD performance for both Terra and Aqua MODIS.

\section{METHODOLOGIES}

Figure 1 illustrates a schematic of the MODIS SDSM Sun view and SD view signals. The SDSM makes alternate observations of direct sunlight through a fixed screen (Sun view) and the sunlight diffusely reflected from the SD (SD view). A rotating mirror is part of the SDSM system, sending light from the Sun view and SD view to the SDSM SIS. After the rotating mirror, the optical path, detectors, and electronics used for the Sun and SD views are identical. In addition, a dark measurement to correct the SDSM detector offset is also performed. The SDSM is typically operated during each SD calibration event, which can happen either with ("closed") or without ("open") the SD screen in front of the SD. The SDSM is operated in one of two different modes, alternate mode or fixed mode. In alternate mode, the Sun view and SD view data are collected during one orbit, with the SDSM's rotating mirror alternating between different views with every scan of the MODIS scan mirror, repeating the sequence: Sun view, dark view, SD view, dark view, and so on. In fixed mode, the Sun view and SD view data are collected separately during two successive orbits. Some SDSM calibrations were performed in fixed mode in the first few years of each mission, but the large majority have been performed in alternate mode. In the following subsections, a brief overview of different approaches applied to track SD degradation is provided.

\section{A. Ratioing Approach}

This is the original approach designed to track the SD degradation using alternate observations of the SD and Sun using the SDSM. From each SD/SDSM calibration event, after appropriate corrections, a ratio of SDSM SD view response to its Sun view response can be computed by

$$
\Delta_{S D}^{i}=\left\langle\frac{d c_{S D}^{i}}{d c_{S U N}^{i}}\right\rangle ; \quad i=1,2, \ldots, 9
$$

where $<>$ denotes an average over all valid scans and all SDSM data samples (three samples are taken during each scan). $d c_{S U N}^{i}$ is the Sun view response of detector $i$ after 
subtracting the dark background response. $d c_{S D}^{i}$ is the SD view response of detector $i$ after subtracting the dark background response, and applying corrections for the viewing angle and SD BRF effects, such that

$$
d c_{S D}^{i}=\frac{D N_{S D}^{i}-\left\langle D N_{d a r k}^{i}\right\rangle}{\cos \theta_{S D} \cdot B R F} ; \quad i=1,2, \ldots, 9
$$

where $\theta_{S D}$ is the SD solar zenith angle, and DN represents the digital counts in SD and dark background. The time series of $\Delta_{S D}^{i}$ (normalized to the first on-orbit SD calibration) provides a measure of the SD degradation at the wavelength of SDSM detector $i$. Note that the SD signal changes with angle in known ways: the BRF dependence and the $\theta_{S D}$ dependence. Eqn.2 shows that the data is corrected for both of these before calculating ratios. There is also a vignetting function (VF) dependence for the "close" data which we don't explicitly correct. Since the LUT we generate is based on the ratio data, it will correct for the combination of the angular dependence of the SUN view and SD view data (including the SD view VF for the close mode data), so we don't have to completely understand the angular dependence of both views independently.

As shown in Figure 1, there is a fixed SDSM screen in the Sun view path. Due to a misalignment of this screen, the projection of the screen pinholes onto the entrance aperture of the SDSM SIS changes with solar viewing angle. This leads to large ripples of about $\pm 5 \%$ in the Sun view responses, $d c_{S U N}$, on both the time scale of one orbit and one year. Because of these ripples, the original ratioing approach could not be used to accurately track the on-orbit changes in SD BRF, especially with observations made over a short period of time early in the mission, when there were not enough years of data to properly characterize the yearly oscillations.

However, after many years of SD/SDSM calibration events, the impact due to SDSM Sun view ripples on the overall trend of the SD degradation time series becomes less significant. In this situation, this original ratioing approach can still be applied. For example, the ratioing measurements could first be averaged in a sliding window average (SWA) over a year, and then fitted over the operational lifetimes. As the window slides forwards with a constant step size, the corresponding time is defined as

$$
t_{k}=T_{0}+(k-1) \delta+\frac{365 n}{2}
$$

where $T_{0}$ presents a reference time (a start point in sliding window), $\delta$ is a step size and $n$ is the number of years in one window. After this pre-processing, a time series of the yearly-averaged ratios can be applied to track the SD BRF degradation.

\section{B. Normalization Approach}

This approach was designed to remove the ripples in the SDSM Sun view responses by normalizing the response of all SDSM detectors to D9. It was the approach used to calibrate MODIS Collection 5 L1B data. It is based on the fact that the ripples recorded by all SDSM detectors, which in principle have the same optical path, are consistent. It also assumes that the degradation at D9 wavelength is small and can be ignored, which was found to be a valid assumption in the early part of the mission $[10,11]$. Specifically, it can be expressed as, 


$$
\left[\Delta_{S D}^{i}\right]_{d 9}=\left\langle\frac{d c_{S D}^{i} / d c_{S D}^{9}}{d c_{S U N}^{i} / d c_{S U N}^{9}}\right\rangle ; \quad i=1,2, \ldots, 8
$$

This normalized ratioing approach can remove the ripples and produce a stable time series to track the SD degradation at other wavelengths $[6,7]$. It is obvious that the performance and effectiveness of this approach strongly depends on the response of the reference detector D9.

\section{LUT Based Approach}

This approach is designed to both reduce the ripples caused due to the SDSM screen artifact (see Eqn. 1) and the impact due to accumulated degradation from the reference detector (D9) in the normalization approach (see Eqn. 4). Instead of directly using the ratios as in Eqn. 1, a look-up-table (LUT) consisting of correction factors which are viewing geometry dependent is formulated to reduce the ripples in the SDSM's Sun view responses. Thus,

$$
\Delta_{S D}^{i}=\left\langle\frac{d c_{S D}^{i}}{d c_{S U N}^{i}} \cdot \Lambda[\alpha, \beta]\right\rangle ; \quad i=1,2, \ldots, 9
$$

where $\Lambda[\alpha, \beta]$ is the correction LUT and $[\alpha, \beta]$ represent the solar elevation and azimuth angles for each scan within a given calibration event. We have developed two different versions of this method: one where all SDSM detectors use the same correction LUT (based on D9), and one where a different LUT is calculated and applied for each detector individually.

This LUT-based method is more attractive as it can efficiently compensate the geometrical artifacts for all SDSM detectors. In the case where we use a D9-based LUT for all detectors, the degradation term Eqn. 1 can be represented by,

$$
\Delta_{S D}^{i}=\left[\Delta_{S D}^{i}\right]_{d 9} \cdot\left(\frac{d c_{S D}^{9}}{d c_{S U N}^{9}} \cdot \Lambda^{9}[\alpha, \beta]\right) ; \quad i=1,2, \ldots, 9
$$

This is the current method used to calibrate MODIS Collection 6 L1B data and Collection 5 L1B data after March 2009. On the other hand, each detector can have its own LUT to compensate the ripple impact (Eqn. 5 , where $\Lambda$ is different for each detector). A dynamic modeling has been developed that generates a LUT for each SDSM detector independently, using calibration data from early mission SDSM observations. The details of the method used to construct the view-geometry-dependent LUT are presented in the next section.

\section{LUT DESIGN AND IMPLEMENTATION}

\section{A. D9 LUT}

In the LUT based approach, the view geometry dependent compensation terms in $\Lambda[\alpha, \beta]$ are of primary interest. As previously stated, $[\alpha, \beta]$ represent the solar elevation and azimuth angles on a per-scan level during the SD calibration. Populating the correction factors in the LUT is equivalent to quantifying the characteristics (variations) introduced by the SDSM attenuation screen shown in Figure 1. The correction factors are derived from about the first three years of SDSM observations, assuming the degradation at the D9 wavelength is extremely small during this time [5]. There are four major steps involved in this process:

STEP-1: Select the SD view and Sun view data for D9 from observations made early in 
the mission (Aqua: 8/31/2002 to $9 / 5 / 2005$; Terra: $2 / 24 / 2000$ to $1 / 6 / 2004)$. The datasets include the SDSM detector responses and the elevation and azimuth angles for every scan in alternate operations mode [8]. To maximize the signal-to-noise ratio, the SD view datasets are taken from the SD screen (SDS) open mode calibration for Aqua. However, for Terra, SDS close mode calibration has to be used as only the close mode is available after the door anomaly in July, 2003.

STEP-2: Define a two-dimensional (2D) array of equally spaced values, covering the relevant angle ranges.

STEP-3: Populate the elements of the LUT $\Lambda[\alpha, \beta]$ from the selected SDSM D9 observations. We choose to define the LUT using the direct ratios:

$$
\Lambda\left[a_{k}, b_{j}\right]=\left\langle\frac{d c_{S U N}^{9}\left[a_{k}, b_{j}\right]}{d c_{S D}^{9}\left[a_{k}, b_{j}\right]}\right\rangle ; \quad a_{k} \in \varpi(\alpha), b_{j} \in \varpi(\beta) \text { (7) }
$$

where $\left[a_{k}, b_{j}\right]$ represents bin indexes with corresponding angles, $\varpi(\alpha)$ and $\varpi(\beta)$, respectively.

STEP-4: Populate missing elements in the LUT. Since only limited datasets are considered, the LUT cannot be populated with all possible angular combinations of the bins, unless an appropriate bin size is used. Consequently, an interpolation strategy needs to be adopted. There are many strategies that could be used to interpolate the available data and fill the missing elements of the LUT. In our method, we first perform a cubic spline interpolation over elevation angle, followed by a nearest-neighbor element weighting, a spline interpolation over azimuth angle, and a local surface smoothing. The details of the method we use to build and populate the D9- based LUT, along with specific examples, were presented in [6].

It is necessary to mention that there are MODIS yaw maneuver measurements, which were designed to cover the entire angular range of solar illumination to the SDS for the entire mission of Terra and Aqua MODIS [10]. Two series of Terra yaw maneuvers were performed in April 2000 and December 2002, respectively. One series of Aqua yaw maneuvers were executed in June 2002. However, yaw data is insufficient to build the LUT. For instance, Figure 2 shows the results of Aqua yaw compared to first-year operation SDSM measurements, in both cases after interpolating the data across elevation angle. Obviously, there is less yaw data compared to the on-orbit data, so the generated LUT will not have sufficient precision. In addition, there are some differences in the shape of the data as a function of elevation angle. For these reasons, we use only the on-orbit SDSM measurements to build the LUT.

\section{B. Detector-dependent Dynamic LUT}

This is an enhanced method to build a LUT for each individual SDSM detector. In the previous subsection, we discussed the methodology to construct the D9 LUT, where a correction factor was developed based on the SDSM D9 ratios of the Sun view to the SD view as a function of elevation and azimuth angle. Here we extend this idea to other SDSM detectors. In principle, the effect of the SDSM screen mis-alignment, and the values of the LUT required to correct for it, should be the same for all detectors. But in practice we find that there are slight detector differences, possibly due to imperfections in the SIS. 
We define and build the individual LUTs using a similar strategy as in the case of D9, however for the other detectors the SD degradation in the early mission is not negligible and we must account for this in the LUT design. All datasets include both SD/Sun view responses and their corresponding elevation and azimuth angles $(\alpha, \beta)$. Then, equally spaced bins of angles $(\alpha, \beta)$ within a pre-defined LUT size (e.g., Aqua LUT 500x500: 500 equally spaced bins for elevation angles in the range of [10.8, 14.3], and 500 for azimuth angles in the range of $[-35,2,-22.0])$ are generated. After that, a LUT for detector $\mathrm{i}, \Lambda^{i}(\alpha, \beta)$, is associated with $\varpi(\alpha)$ and $\varpi(\beta)$, digitized $(\alpha, \beta)$ angles. Assume that $\left[a_{k}, b_{j}\right]$ denotes joint bin indexes, then we have,

$$
\begin{aligned}
\Lambda^{i}\left(a_{k}, b_{j}\right) & =\left\langle\frac{d c_{S U N}^{i}\left[a_{k}, b_{j}\right]}{d c_{S D}^{i}\left[a_{k}, b_{j}\right]} \cdot \eta^{i}\right\rangle \\
a_{k} & \in \varpi(\alpha), b_{j} \in \varpi(\beta), i=1,2, \ldots, 9
\end{aligned}
$$

where $\eta^{i}$ is the reciprocal of degradation on detector $i$ at the time SDSM data considered. Elements of the LUT are populated with data from early mission (the precise data is defined below). Then, missing elements are filled by performing a spline interpolation over elevation and azimuth dimensions, as well as a local smooth function. Using these LUTs defined for each detector, the SDSM detector degradation can be calculated by,

$$
\Delta_{S D}^{i}=\left\langle\frac{d c_{S D}^{i}(\alpha, \beta)}{d c_{S U N}^{i}(\alpha, \beta)} \cdot \Lambda^{i}(\alpha, \beta)\right\rangle ; i=1,2, \ldots, 9 .
$$

Since the per-detector LUT is generated using the first few years of SD and Sun observations, the degradation of the SD in this time-period cannot be ignored. In order to mitigate this effect, a dynamic LUT approach has been formulated. Figure 3 illustrates the dynamic scheme used to build LUTs for each individual detector. At first, SDSM raw data are corrected using the initial screen compensation LUT, where the previously generated D9-based LUT is used. Then, degradation estimation is performed for each detector. The degradation coefficient of each detector will be re-applied as a part of the de-trending process. After de-trending, the processed SDSM data will be used for each individual detector LUT re-generation. Each detector's degradation is then reestimated using this re-generated LUT. The dynamic routine is iterated until a convergence of the fitting residue is obtained. In general, there are four steps described below:

STEP-1: Establish a LUT using about the first five years of SD and SUN raw response, assuming no D9 degradation involved (details in the next section).

STEP-2: Apply the established LUT to all available data from each detector, and get each individual detector degradation estimate using all the corrected data.

STEP-3: Re-build individual LUT for each detector using five-year data with the detrending process, where the estimated degradation is corrected for each detector.

STEP-4: If the difference between previous/current degradation estimates is less than a predefined value, then stop, otherwise go to STEP-2 to get a new degradation iteratively. The dynamic results show that five iterations are sufficient to obtain a stable estimate (degradation values on successive iterations within $0.1 \%$ ) for the SDSM screen characterization.

IV. DYNAMIC LUT COMPARISON 


\section{A. Aqua Dynamic LUT}

The first five years of SDSM alternate mode measurements are used to derive the LUT. To eliminate the uncertainty associated with screened SD measurements, only the data acquired with the SD screen open are used to build the LUT for Aqua.

We next compare the resulting LUTs from different detectors, in order to examine the impact of using a separate LUT for each detector, rather than the same D9-based LUT. Since the SDSM detector signals have different magnitudes, a normalization process is needed to compare LUTs between different detectors. Suppose a LUT has $N \times N$ elements, from Eqn. 8, we have a normalization factor for each detector:

$$
\begin{aligned}
\zeta_{i}= & \frac{N^{2}}{\sum_{k=0}^{N-1} \sum_{j=0}^{N-1} \Lambda^{i}\left(a_{k}, b_{j}\right)} \\
& a_{k} \in \varpi(\alpha), b_{j} \in \varpi(\beta), i=1,2, \ldots, 9 .
\end{aligned}
$$

Figure 4 shows a three-dimensional profile of the Aqua D9 LUT after normalization, and also shows contour plots representing the difference between the normalized LUTs of three other detectors (D1, D3 and D6) and the D9 LUT, expressed by

$$
\delta_{i}=\Lambda^{i}(\alpha, \beta)-\Lambda^{9}(\alpha, \beta) ; i=1,2, \ldots, 8 .
$$

The Aqua D9 LUT surface profile plotted as a function of elevation and azimuth angles shows fluctuations that are reflective of the SDSM screen's attenuation. The magnitude of the differences for the contour plots are given by the color bar, which is shown in the right lower corner. From the comparison results, a difference of up to $2 \%$ is observed in D1. The LUT's, except for those of D1-D3, closely match the D9 LUT. To present the LUT features associated with the elevation angles, Figure 5 plots the LUTs for all Aqua detectors for four representative azimuth angles of $-33.48^{\circ},-39.45^{\circ},-27.43^{\circ}$ and $24.40^{\circ}$. The elevation profiles of the LUT clearly have different features depending on the azimuth angles.

\section{B. Terra Dynamic LUT}

In the case of Terra MODIS, there are two distinctive trends in the SD degradation: before and after the July 2003 SD door (SDD) anomaly [2-4]. After the door anomaly, the SDD is in a fixed open position and the SDS is permanently in place so that all the RSB are always calibrated using an attenuated solar response. As a result of this operational change, Terra MODIS RSB calibration coefficients can be monitored every orbit. The degradation rates have accelerated after this anomaly, and are strongly wavelength dependent with larger degradation observed at short wavelengths. After the anomaly, the SDSM measurement is only available with the SDS in place. For consistency, only SDS close mode data are used to build the LUT. In practice, the measurements from $2 / 24 / 2000$ to $1 / 6 / 2004$ are used to build an initial LUT for each detector, and then dynamic iterations are performed on the 5-year data after the SDD anomaly (7/2/2003 to 7/8/2008).

Similar to the case of Aqua MODIS, Figure 6 shows the Terra D9 LUT surface profile and difference contours for three other detectors (D1, D3 and D6) relative to it. The difference is in the range of $-3 \%$ to $4 \%$, which is larger than Aqua MODIS. The reason is mainly due to the fact that Aqua uses SDS "open-mode" data and Terra uses SDS "close-mode" data, which has higher noise. Figure 7 plots LUTs for all Terra detectors for 
four azimuth angles of $-30.30^{\circ},-27.06^{\circ}$, $23.82^{\circ}$ and $-20.58^{\circ}$. Similar to Aqua, the Terra LUT shows different behavior for different azimuth angles as well. The differences could be mainly due to imperfections in the SIS and/or the detector alignment in the SIS so that not all detectors see the identical signal.

\section{Estimation Comparisons}

In the previous sub-sections, two methods are discussed to build the correction LUT, either using D9 only or using a dynamic model for each SDSM detector. Here, we will show that SD degradation can be much more reliably derived if the Sun view screen pinhole effects are characterized using the detector-based dynamic model. Although the LUT is applied to all the SDSM detectors, only the results from select detectors are presented for clarity. A comparison of three datasets are presented in the two charts in Figure 8: (i) raw ratios of measurements in the SUN view and the SD view; (ii) corrected ratios using a D9-based LUT, which is currently employed in the MODIS Collection 6 algorithm; (iii) corrected ratios using the newly proposed dynamic individual LUTs. In case (ii), the D9-based LUT is constructed using the early mission data without detrending, as D9 degradation in the first three years is negligible. In the current C6 L1B algorithm, the corrected ratios are further processed by using a 1-year SWA only for SDSM D9. Based on Figure 8, we can observe that the case of using the dynamic individual LUTs provides the best result, where much smaller fluctuations in the data trending can be achieved. In addition, using the SWA is not necessary as the data behavior is stable.
The shorter wavelength detectors, such as D1 and D2, show larger SD degradation. The three data-sets are fitted using a common fitting scheme and the comparison between the results obtained from case (ii) and case (iii) is shown in Figure 9. Considering all SDSM detectors, the fitting difference is less than $+/-1 \%$ for Terra, and $0.3 \%$ for Aqua, respectively. The fitting residues of Terra SDSM D1-D8 in the two cases are plotted in Figure 10, where the red diamonds indicate the results of using dynamic individual LUTs, and the blue crosses indicate the results using only the degradation normalized to D9 (not including the D9 degradation). Smaller residues are observed for all 8 detectors in the dynamic individual LUTs. Similarly, we plot the fitting residues for Aqua in Figure 11. Although the fitting results of D7 and D8 show a little larger residues in the case of Aqua dynamic individual LUTs, the estimation standard deviation (STD) of the total degradation could still be better, since in case (ii) the total degradation includes the normalized degradation times the D9 degradation. Specifically, in the case where we use a LUT for D9 only and the other detectors are normalized to D9, let $\left\{\sigma_{i}\right\}$ represent the STD of the normalized degradation for SDSM detector $i$. The combined STD for the 8 detectors will be

$\sigma_{i}^{\prime}=\sqrt{\sigma_{i}^{2}+\sigma_{9}^{2}} ; \quad i=1,2, \ldots, 8$.

Figure 12 presents Terra and Aqua estimation standard deviation using the two methods. Since Terra SDSM uses "close" mode data, its deviation level is higher than that of Aqua SDSM. The two different methods are similar for D9, however the use of dynamic iterations and a different input data range results in a slightly improved STD for D9 in the individual-detector method. Also, the individual-detector method 
provides better result for the other 8 detectors. Having data with smaller standard deviation is important, as it makes it easier to identify changes in trends and make forward predictions. It is necessary to mention that the MODIS uncertainty algorithm includes the uncertainty of the SD calibration in a detailed way which has been documented before [13]. The uncertainty in calculating the SDSM degradation ratio is just one part of the uncertainty calculation. The uncertainty in the SDSM calibration does not change dramatically over the mission. The main impact of the SD degradation to the MODIS uncertainty is the decreasing SNR of the $\mathrm{RSB}$, however this remains above the instrument specification for all RSB bands [14].

\section{MODIS SDSM AND SD PERFORMANCE}

In the previous sections, we presented methods to effectively monitor MODIS SD degradation using SDSM measurements. Comparison studies reveal that both LUT based approaches provide consistent results for both MODIS Terra and Aqua. Terra difference is within $\pm 0.85 \%$, and Aqua is within $\pm 0.18 \%$ for all detectors. On the other hand, if limited data is available, the original ratioing approach with normalization to D9 is necessary. The normalization approach works fine as long as D9 degradation is negligible. For high quality monitoring of changes in SD BRF over a long-term time series, additional corrections are necessary to remove the impact due to the D9 degradation. The LUT based model is more effective as it efficiently compensates for the geometrical impacts.

\section{A. MODIS SDSM Results}

The SD degradation is due to polymerization of contaminants in the SD. Thus, the longer the exposure to sunlight, the more degradation will be observed. In order to prolong the operational lifetime of the SD and SDSM, the frequency of the calibration activities has been gradually reduced. During the early mission, SD/SDSM calibrations were performed for both sensors on a daily or weekly basis to track SD BRF change. Over time, the frequency was reduced to two weeks and subsequently to three weeks, which is the current operational configuration. Now, the SD is operated with SDS open configuration once every six weeks for Aqua MODIS, although the SDSM operation still remains on a tri-weekly basis. Fortunately, the SD degradation does not exhibit any dependence on the SD/SDSM calibration frequency. The reason is that in addition to SD calibration, the SD also degrades due to exposure to scattered light through the nadir port during the daylight part of each orbit. Data analysis [10] shows that nadir scattered light dominates the SD degradation, as compared to direct illumination during the calibration itself, especially for the three short-wavelength MODIS bands, band-8 $(0.412 \mu \mathrm{m})$, band-9 $(0.443 \mu \mathrm{m})$ and band-3 $(0.469 \mu \mathrm{m})$.

Using the dynamic LUTs, Figure 13 plots Terra and Aqua MODIS degradation trending results, where all SDSM detectors use the same fitting strategy. Notice that the Aqua MODIS SDSM degradation rates are nearly identical to Terra when both instruments are operated under the same conditions. Thus, in the first three years of operation, Aqua and Terra have similar degradation profiles. In the case of MODIS Terra, there are two distinctive trends in the SD degradation: before and after the SD door (SDD) anomaly in May 2003. The degradation rates have 
accelerated after this anomaly due to increased solar exposure of the SD, and are strongly wavelength dependent with larger degradation observed at shorter wavelength. Also, it was found that in 2014-2015, the trends in SD degradation reversed direction in both instruments for a brief period. The reason for this coincident change in the trend is currently unclear. Table 2 summarizes the results for Aqua/Terra SDSM D1 to D9, including total degradation rates and estimation standard deviation.

\section{B. MODIS SD Performance}

After MODIS Aqua/Terra degradation profiles are characterized over all nine SDSM detectors, the overall SD performance of MODIS Aqua and Terra can be evaluated. Using the relationship between SDSM detectors and RSB bands, and a linear interpolation approximation, the MODIS SD degradation versus center wavelength can be obtained. Figure 14 plots their yearly degradation performance. Notice that the degradation is somewhat saturated in both instruments. The degradation change in the last 4 years is about $1.0 \%$ for Aqua, and $1.2 \%$ in the last 6 years for Terra. Specifically, operating after about seventeen years of operation, the Terra MODIS SD BRF has a wavelength dependent degradation of $47.7 \%$ at $0.412 \mu \mathrm{m}, 36.0 \%$ at $0.466 \mu \mathrm{m}, 25.7 \%$ at $0.530 \mu \mathrm{m}, 21.5 \%$ at $0.554 \mu \mathrm{m}, 12.5 \%$ at 0.646 $\mu \mathrm{m}, 6.6 \%$ at $0.747 \mu \mathrm{m}, 3.3 \%$ at $0.857 \mu \mathrm{m}$, $1.4 \%$ at $0.904 \mu \mathrm{m}$ and $1.4 \%$ at $0.936 \mu \mathrm{m}$. Meanwhile, in over fifteen years of operation, the degradation profile of Aqua MODIS SD BRF is $18.5 \%$ at $0.412 \mu \mathrm{m}$, $12.2 \%$ at $0.466 \mu \mathrm{m}, 7.5 \%$ at $0.530 \mu \mathrm{m}, 6.2 \%$ at $0.554 \mu \mathrm{m}, 3.3 \%$ at $0.646 \mu \mathrm{m}, 1.7 \%$ at 0.747 $\mu \mathrm{m}, 0.9 \%$ at $0.857 \mu \mathrm{m}, 0.6 \%$ at $0.904 \mu \mathrm{m}$ and $0.6 \%$ at $0.936 \mu \mathrm{m}$.

\section{CONCLUDING REMARKS}

In this paper, we developed a LUT based approach to effectively track the MODIS SD degradation based on SDSM observations. A recent improvement of MODIS SD on-orbit degradation characterization has been presented. A LUT-based approach, initially applied only to SDSM D9, is now extended to other SDSM detectors using a dynamic scheme. The overall on-orbit performance of Aqua and Terra MODIS SD/SDSM has been evaluated. By applying the LUT geometrical compensation, the SDSM observations can be consistently used to track the on-orbit changes of the SD BRF, such that high quality RSB calibration and science data quality are maintained. To combat the impact of SDSM Sun view ripples, different approaches have been discussed to track MODIS SD on-orbit degradation. Compared with the raw SDSM measurements, a dynamic LUT compensation out-performs the other options in terms of observed fluctuations and will be considered for implementation in the future versions of the MODIS L1B. Results of the SD degradation derived from SDSM measurements are presented and compared. In the yearly-based consideration on the shortest wavelength band, the Aqua SD degrades about $19 \%$ at the center wavelength of $0.412 \mu \mathrm{m}$ in 15 years; however the Terra SD degrades about $48 \%$ in 17 years.

\section{ACKNOWLEDGMENT}

The authors would like to thank other members of the MODIS Characterization Support Team (MCST) for their technical assistance and discussions during this study. Particularly, the authors wish to acknowledge Dr. Truman Wilson for his valuable comments and useful suggestions. 


\section{REFERENCES}

[1] W. L. Barnes and V. V. Salomonson, "MODIS: A Global Image Spectroradiometer for the Earth Observing System", Critical Reviews of Optical Science and Technology, CR47, 285-307, 1993.

[2] W. L. Barnes, X. Xiong and V. V. Salomonson, "Status of Terra MODIS and Aqua MODIS:, Journal of Advances in Space Research, 32/11, 2099-2106, 2003.

[3] Xiong, X., J. Sun, W. Barnes, V. Salomonson, J. Esposito, H. Erives and B. Guenther, "Multiyear On-Orbit Calibration and Performance of Terra MODIS Reflective Solar Bands", IEEE Trans. on Geosci. Remote Sens., 45(4), 879-889, 2007

[4] J. Sun, X. Xiong and W.L. Barnes, "MODIS Solar Diffuser Stability Monitor Sun View Modeling", IEEE Trans. on Geosci. Remote Sens., 43(8), 1845-1854, 2005

[5] X. Xiong, H. Erives, S. Xiong, X. Xie, J. Esposito, J. Sun and W. Barnes, "Performance of Terra MODIS Solar Diffuser and Solar Diffuser Stability Monitor", proceedings of SPIE - Earth Observing Systems X, Vol. 5882, 2005.

[6] H. Chen, Z. Wang, J. Sun, A. Angal and X. Xiong, "MODIS Solar Diffuser On-orbit Degradation Characterization Using Improved SDSM Screen Modeling", proceedings of SPIE - Earth Observing Systems XVII, Vol. 9998, 2016.

[7] H. Chen, Z. Wang, J. Sun, A. Angal and X. Xiong, "Recent Progress of MODIS Solar Diffuser On-orbit Degradation Characterization", proceedings of SPIE -
Earth Observing Systems XVII, Vol. 8510, 2012.

[8] H. Chen and X. Xiong, "MODIS Solar Diffuser Monitor: Function and Applications", proceedings of SPIE - Earth Observing Systems XIV, Vol. 7452, 2009.

[9] J. Sun, X. Xiong and B. Guenther, "MODIS Solar Diffuser Stability Monitor Performance", proceedings of SPIE - Earth Observing Systems VI, Vol. 4483, 156-164, 2002.

[10] X. Xiong, X. Xie, A. Angal, J. Choi, J. Sun and W. Barnes, "Characterization of MODIS Solar Diffuser On-orbit Degradation", proceedings of SPIE - Earth Observing Systems XII, Vol. 6677, 2007.

[11]X. Xiong, and W.L. Barnes, "An Overview of MODIS radiometric calibration and characterization", $A d v$. Atmos. Sci., 23(1), 69-79, 2006.

[12] C Cao, X. Xiong, A. Wu and X. Wu, "Assessing the consistency of AVHRR and MODIS L1B reflectance for generating Fundamental Climate Data Records", Journal of Geophysical Research, Vol.113, D09114, 2008.

[13] X. Xiong, J. Sun, A. Wu, K.-F. Chiang, J. Esposito, and W. Barnes, "Terra and Aqua MODIS calibration algorithms and uncertainty analysis," Proceedings of SPIE Vol. 5978, p.59780V, 2005.

[14] A. Angal, X. Xiong, J. Sun, and X. Geng, "On-orbit noise characterization of MODIS reflective solar bands," Journal of Applied Remote Sensing, vol. 9, no. 1, p. 094092, 2015. 
TABle 1 Center Wavelengths of SDSM DEtectors ANd Matching MODIS BandS

\begin{tabular}{cc|cc}
\hline SDSM Detectors & CW & MODIS Bands & CW \\
\hline D1 & 0.412 & 8 & 0.412 \\
D2 & 0.466 & 3 & 0.469 \\
D3 & 0.530 & 11 & 0.531 \\
D4 & 0.554 & 4 & 0.555 \\
D5 & 0.646 & 1 & 0.645 \\
D6 & 0.747 & 15 & 0.748 \\
D7 & 0.857 & 2 & 0.858 \\
D8 & 0.904 & 17 & 0.905 \\
D9 & 0.936 & 18 & 0.936 \\
\hline
\end{tabular}

$\mathrm{CW}$ : Center Wavelengths in $\mu \mathrm{m}$ 
Table 2 MOdis Aqua and Terra SD Degradation Performance

\begin{tabular}{|c|c|c|c|c|c|c|c|}
\hline & & & Aqua & & Terra & & \\
\hline $\begin{array}{l}\text { SDSM } \\
\text { detector }\end{array}$ & $\begin{array}{l}\text { Wavelength } \\
(\mu \mathrm{m})\end{array}$ & \begin{tabular}{|l|} 
MODIS \\
bands
\end{tabular} & $\begin{array}{l}\text { Degradation } \\
\text { (2016242) }\end{array}$ & STD (\%) & $\begin{array}{l}\text { Degradation I } \\
(\mathbf{2 0 0 3 1 8 2})\end{array}$ & $\begin{array}{l}\text { Degradation II } \\
(2016250)\end{array}$ & STD (\%) \\
\hline D1 & 0.41 & 8 & 0.8218 & 0.24 & 0.9009 & $\mathbf{0 . 5 0 3 7}$ & \begin{tabular}{|l|}
0.48 \\
\end{tabular} \\
\hline D2 & 0.46 & 3 & 0.8824 & 0.20 & 0.9358 & 0.6148 & \begin{tabular}{|l|l|}
0.42 \\
\end{tabular} \\
\hline D3 & 0.53 & 11 & 0.9270 & 0.19 & 0.9598 & 0.7262 & 0.45 \\
\hline D4 & 0.55 & 4 & 0.9396 & 0.19 & 0.9667 & 0.7656 & 0.39 \\
\hline D5 & 0.65 & 1 & 0.9676 & 0.18 & 0.9797 & 0.8634 & 0.40 \\
\hline D6 & 0.75 & 15 & 0.9835 & 0.17 & 0.9887 & 0.9280 & 0.42 \\
\hline D7 & 0.86 & 2 & 0.9908 & 0.17 & 0.9957 & 0.9661 & 0.43 \\
\hline D8 & 0.90 & 17 & 0.9938 & 0.16 & 0.9977 & 0.9873 & \begin{tabular}{|l|}
0.43 \\
\end{tabular} \\
\hline D9 & 0.94 & 19 & 0.9966 & 0.18 & 0.9977 & 0.9884 & 0.42 \\
\hline
\end{tabular}




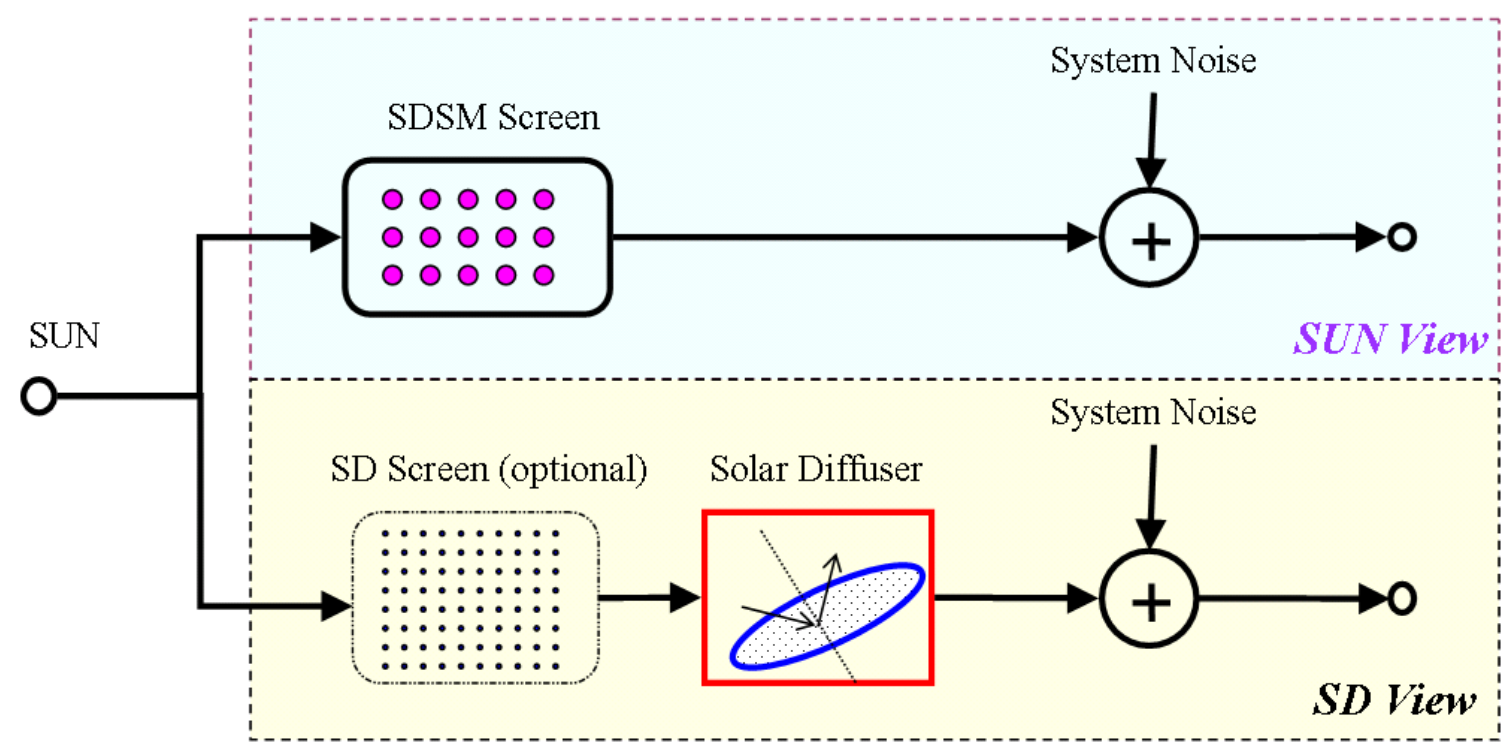

Figure 1 SDSM SUN view and SD view (sunlight diffusely reflected from the SD). 

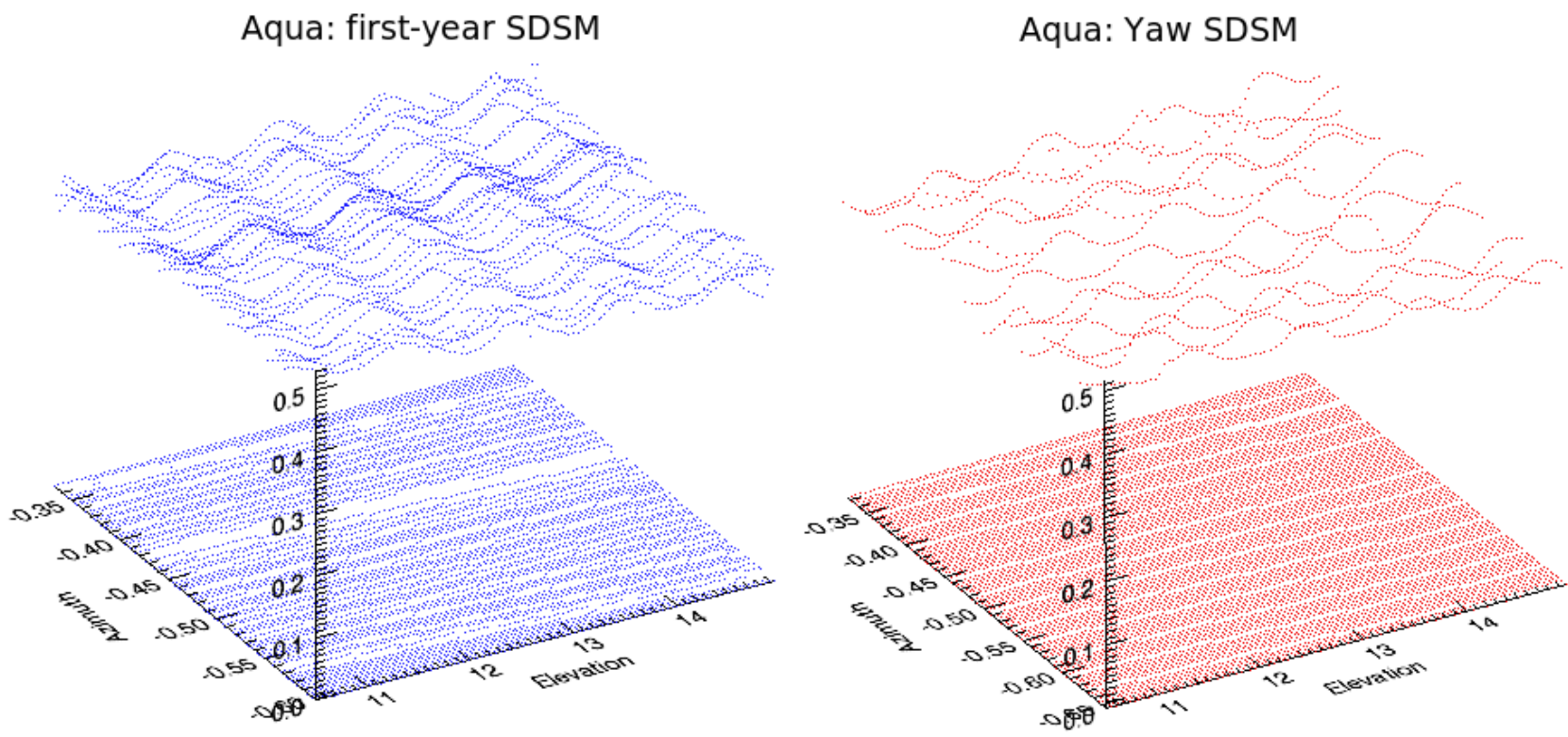

Figure 2 SDSM ratio values (SD view / Sun view) for the first year of Aqua regular on-orbit SDSM calibrations (left panel) and for the Aqua yaw maneuvers (right panel). In both cases, the data within one orbit has been interpolated across elevation angle. 


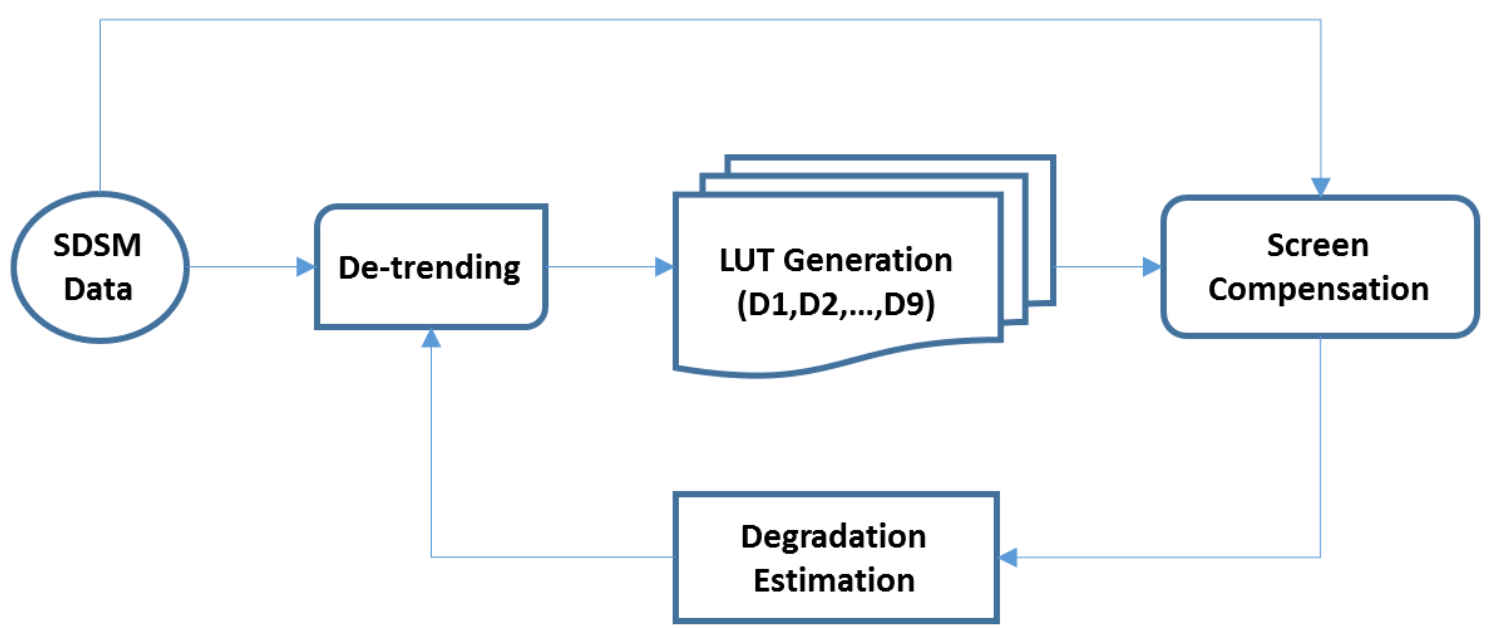

Figure 3 A dynamic scheme to build the detector independent LUT. 


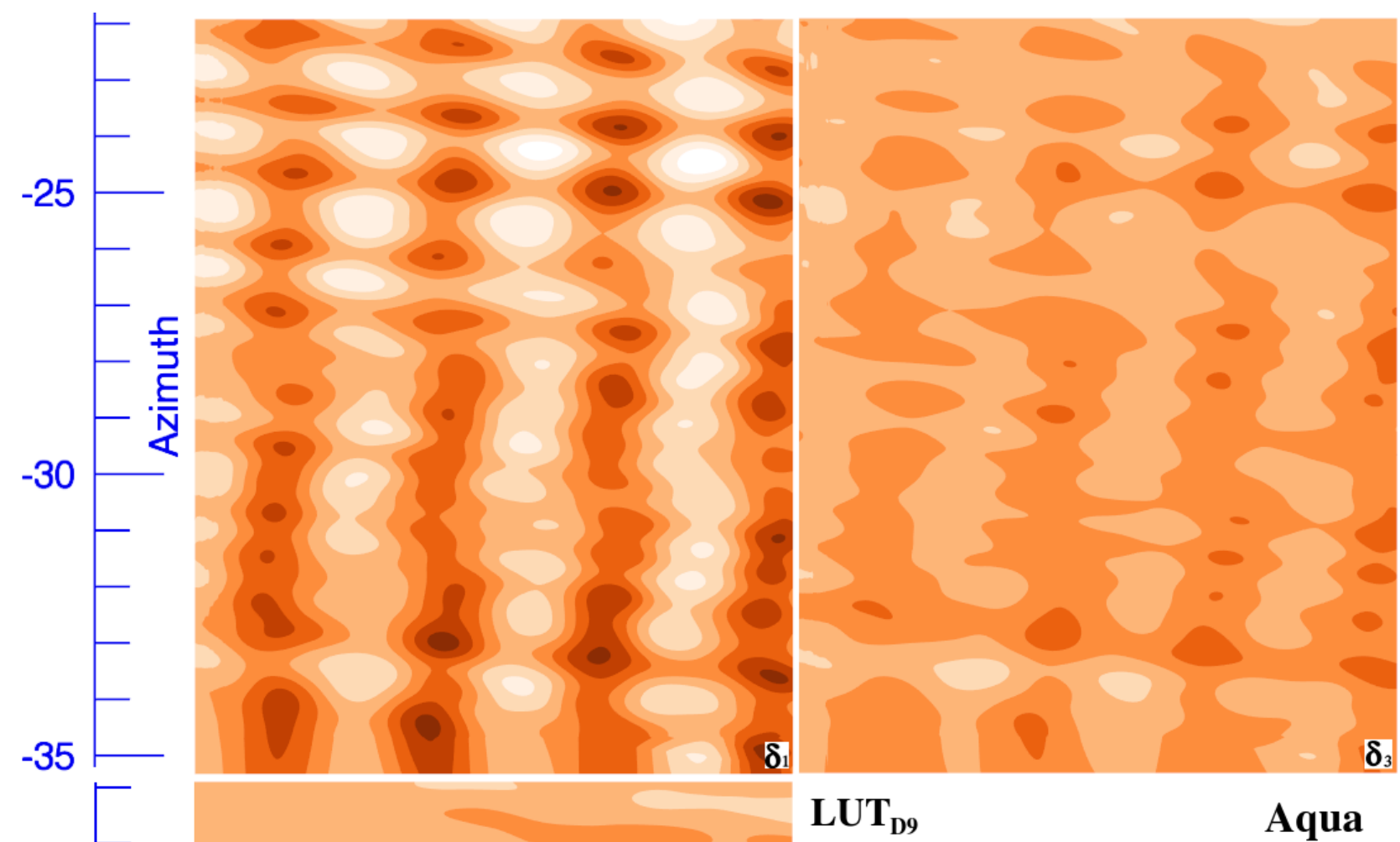



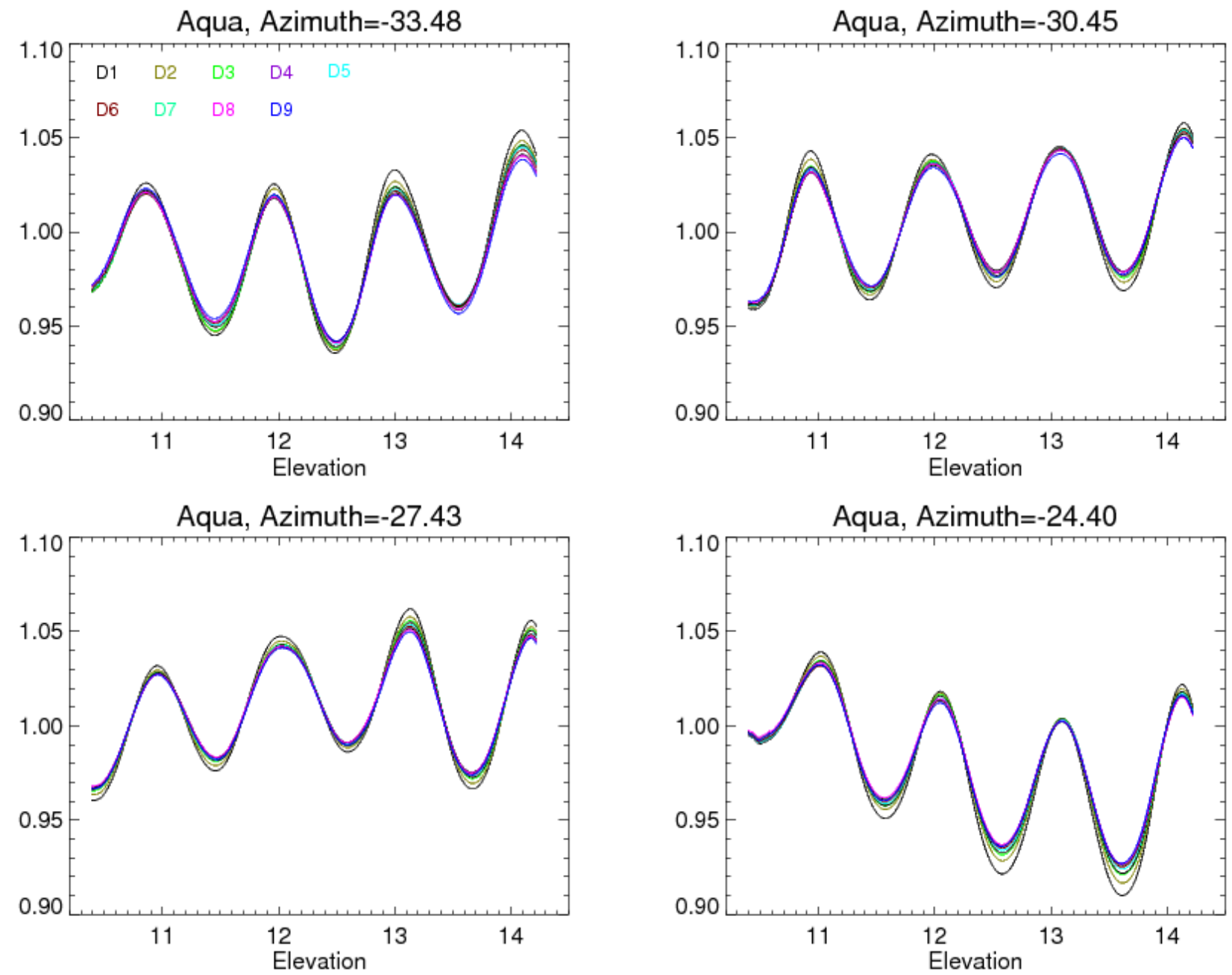

Figure 5 Aqua SDSM pin-hole screen elevation profiles for all detectors in four azimuth angles. 


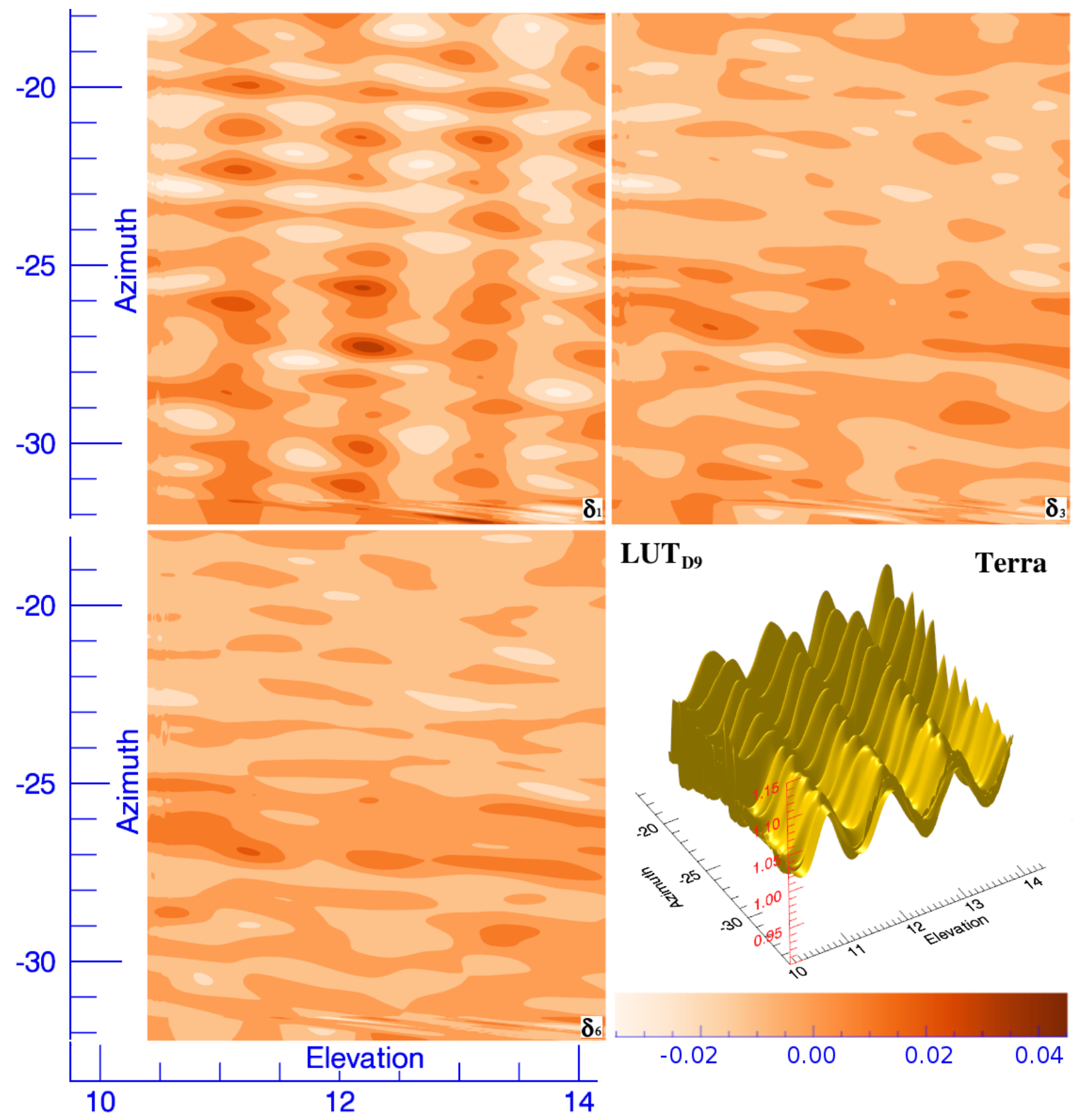

Figure 6 Terra dynamic LUT using first five-years SDSM measurements after the SDD anomaly. 

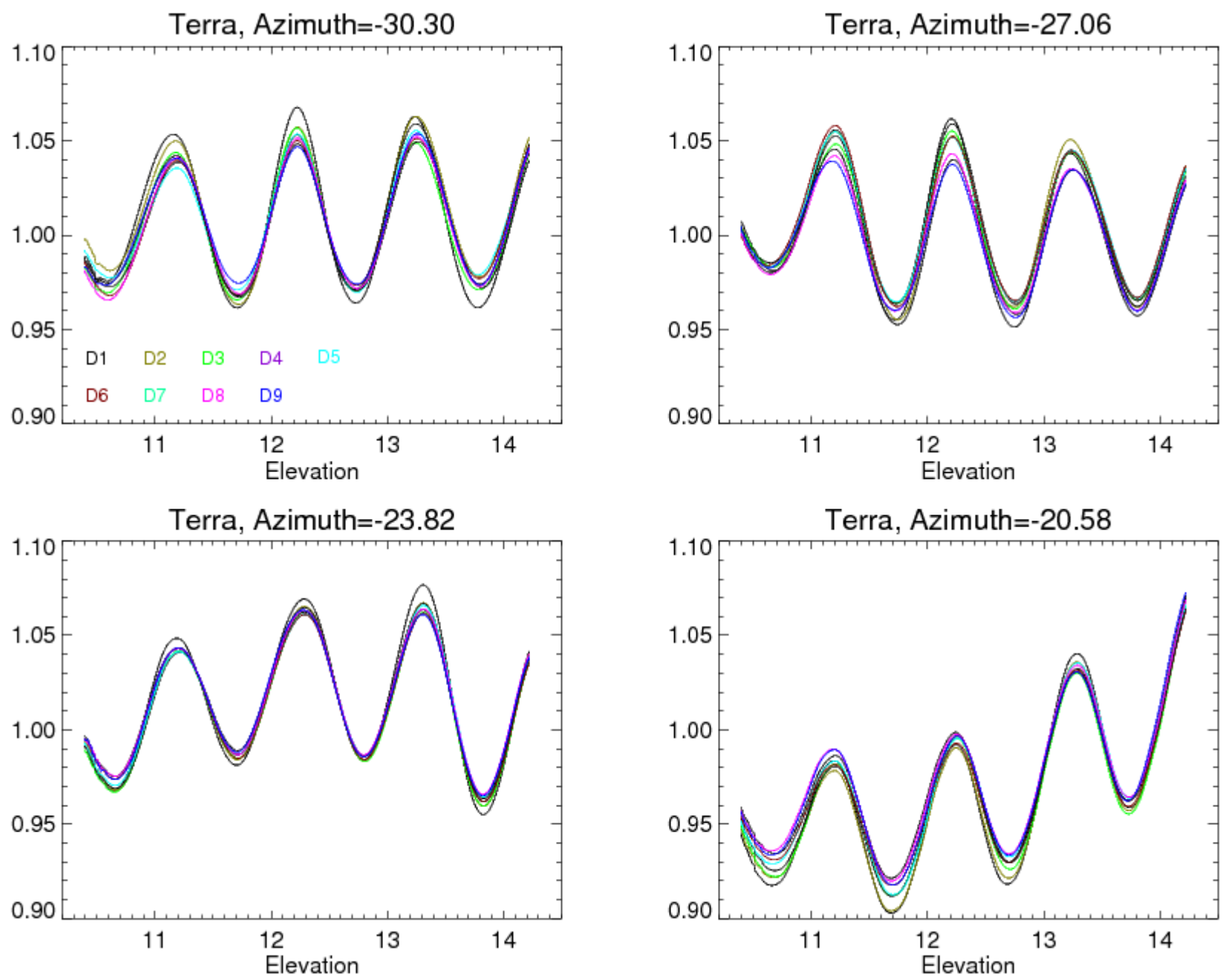

Figure 7 Terra SDSM pin-hole screen elevation profiles for all detectors in four azimuth angles. 

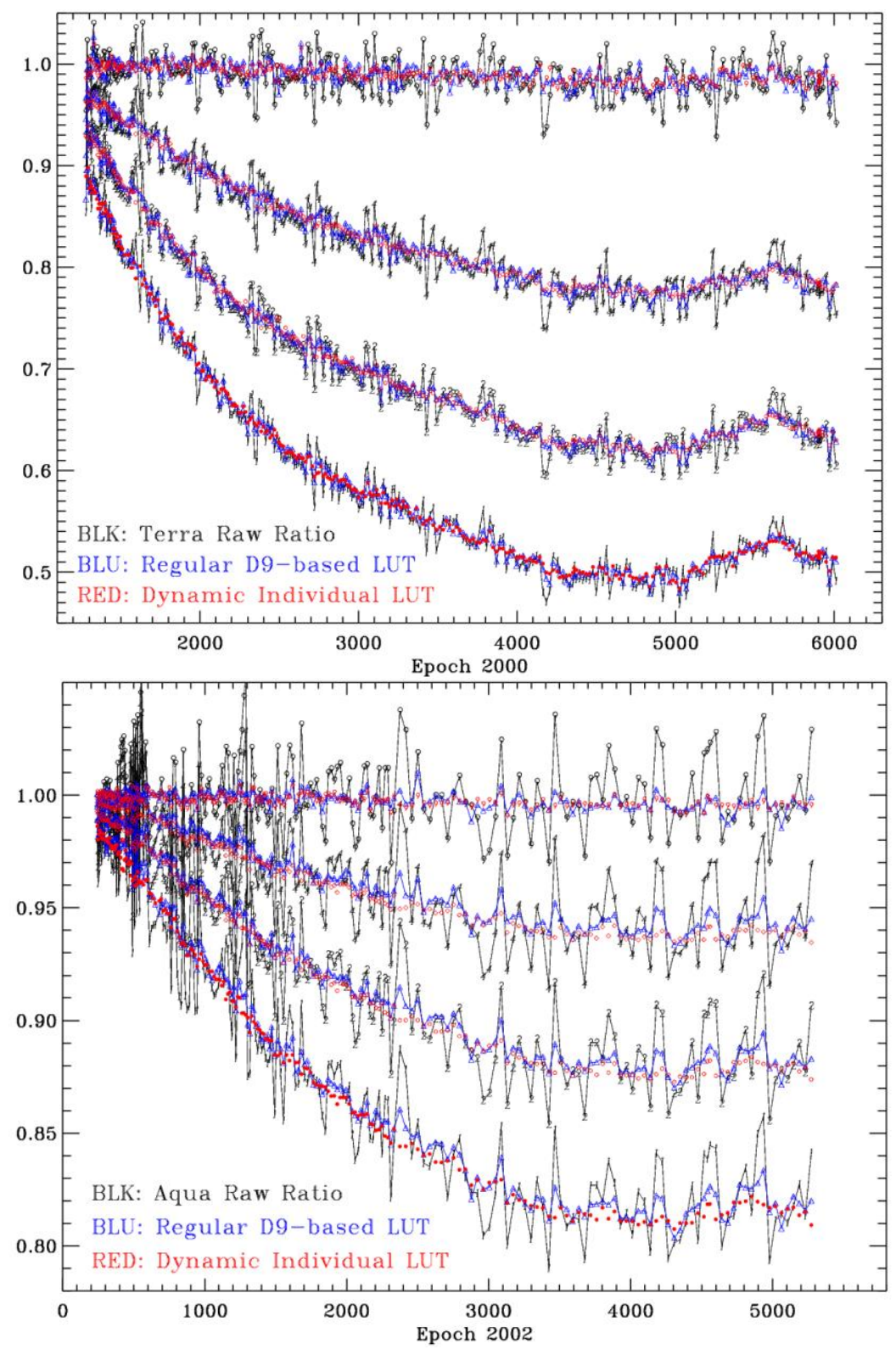

Figure 8 Terra and Aqua SDSM performance trending of four detectors (D1, D2, D4, D9). 

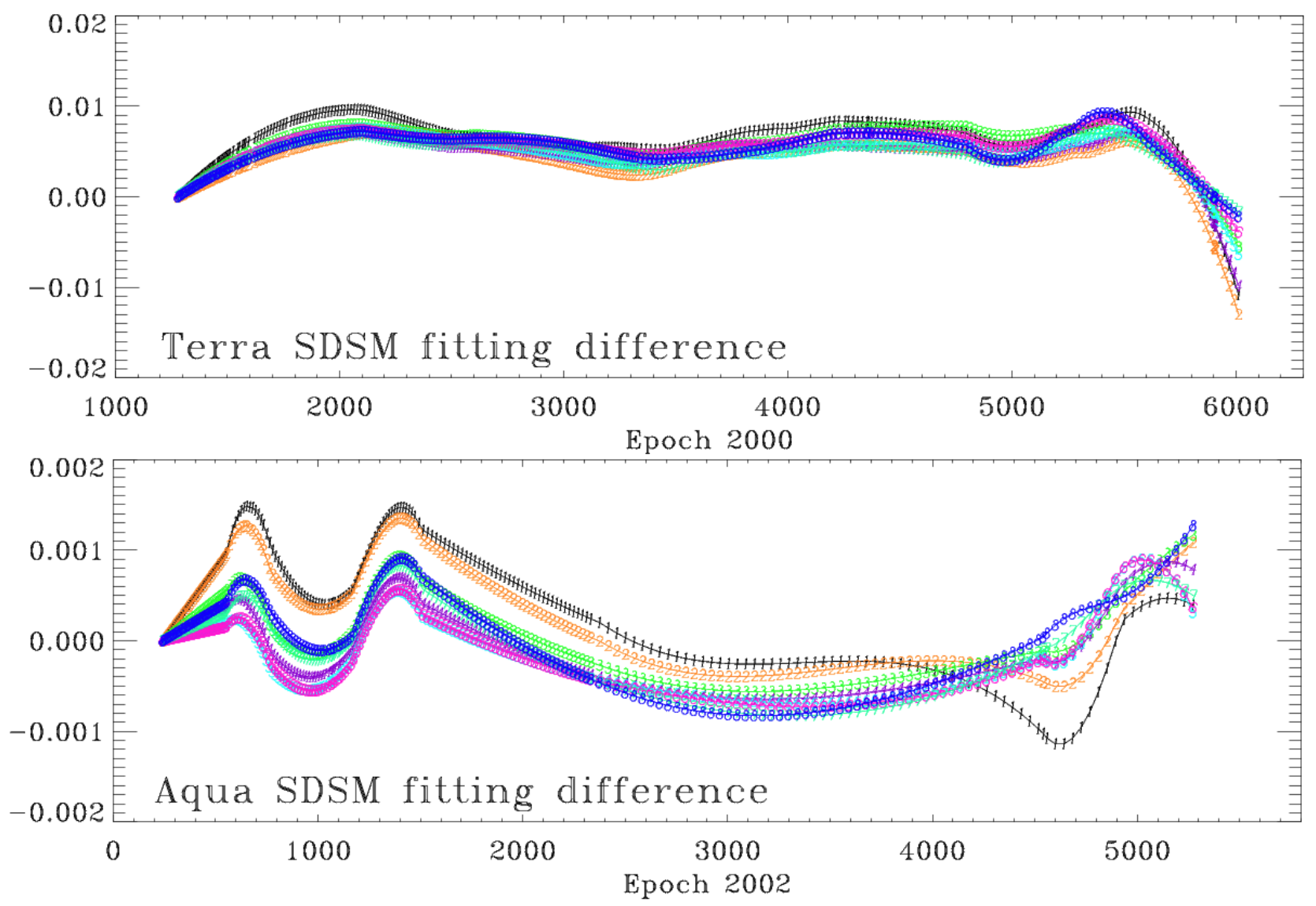

Figure 9 Terra and Aqua SDSM fitting difference between two LUT-based methods for D1-D8. 

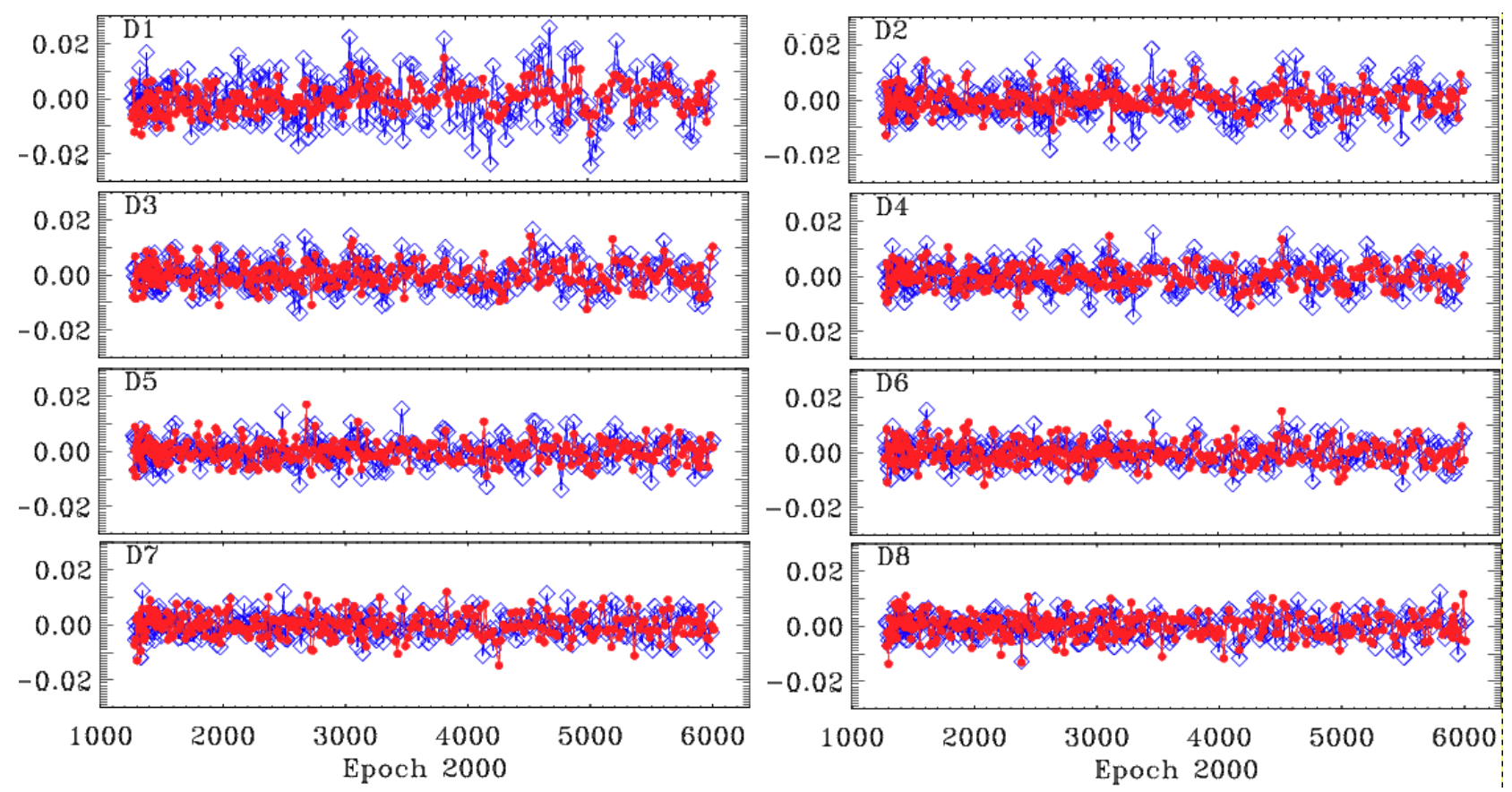

Figure 10 Terra SDSM fitting residues in two LUT-based method for D1-D8. 

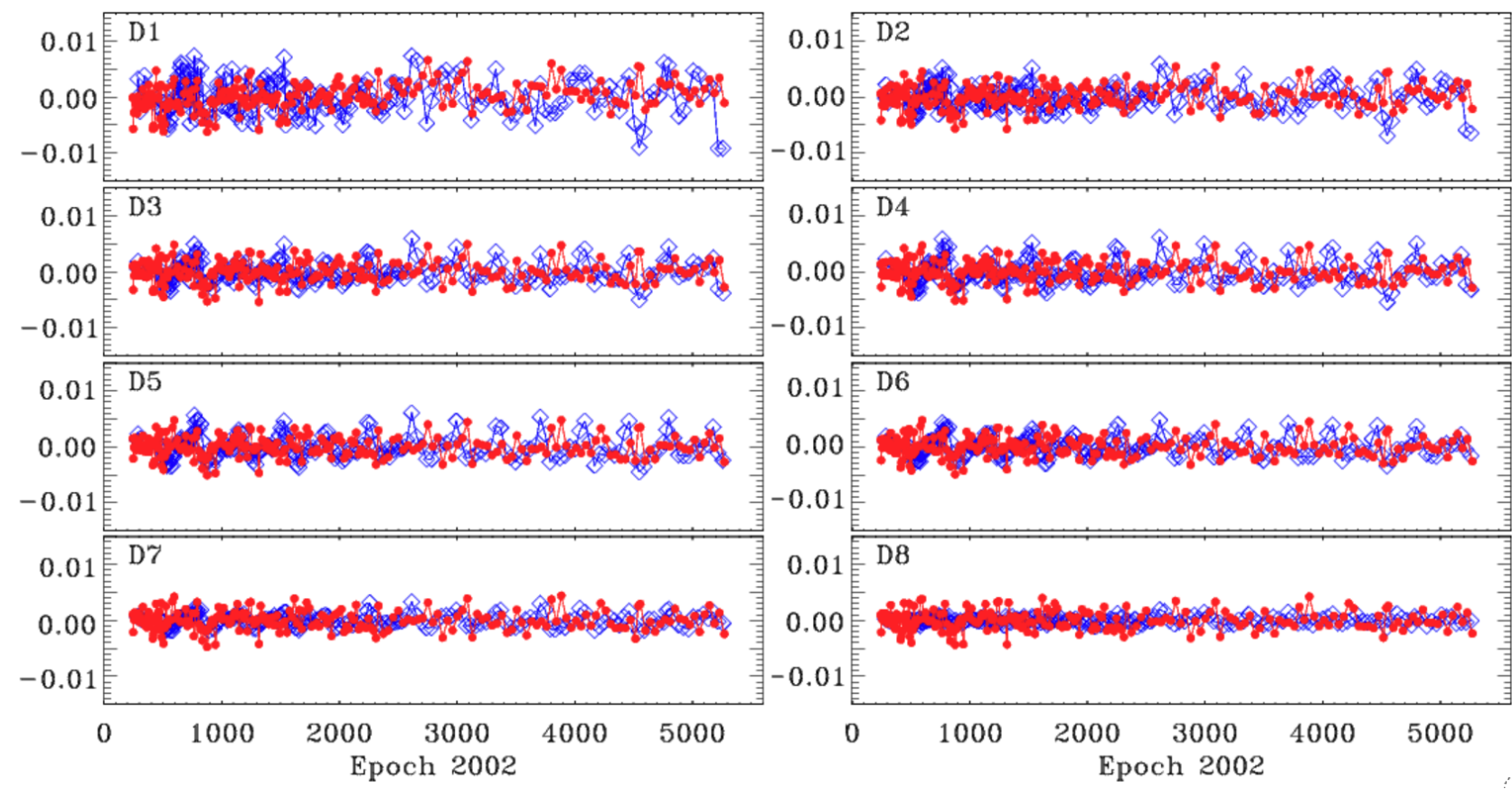

Figure 11 Aqua SDSM fitting residues in two LUT-based method for D1-D8. 


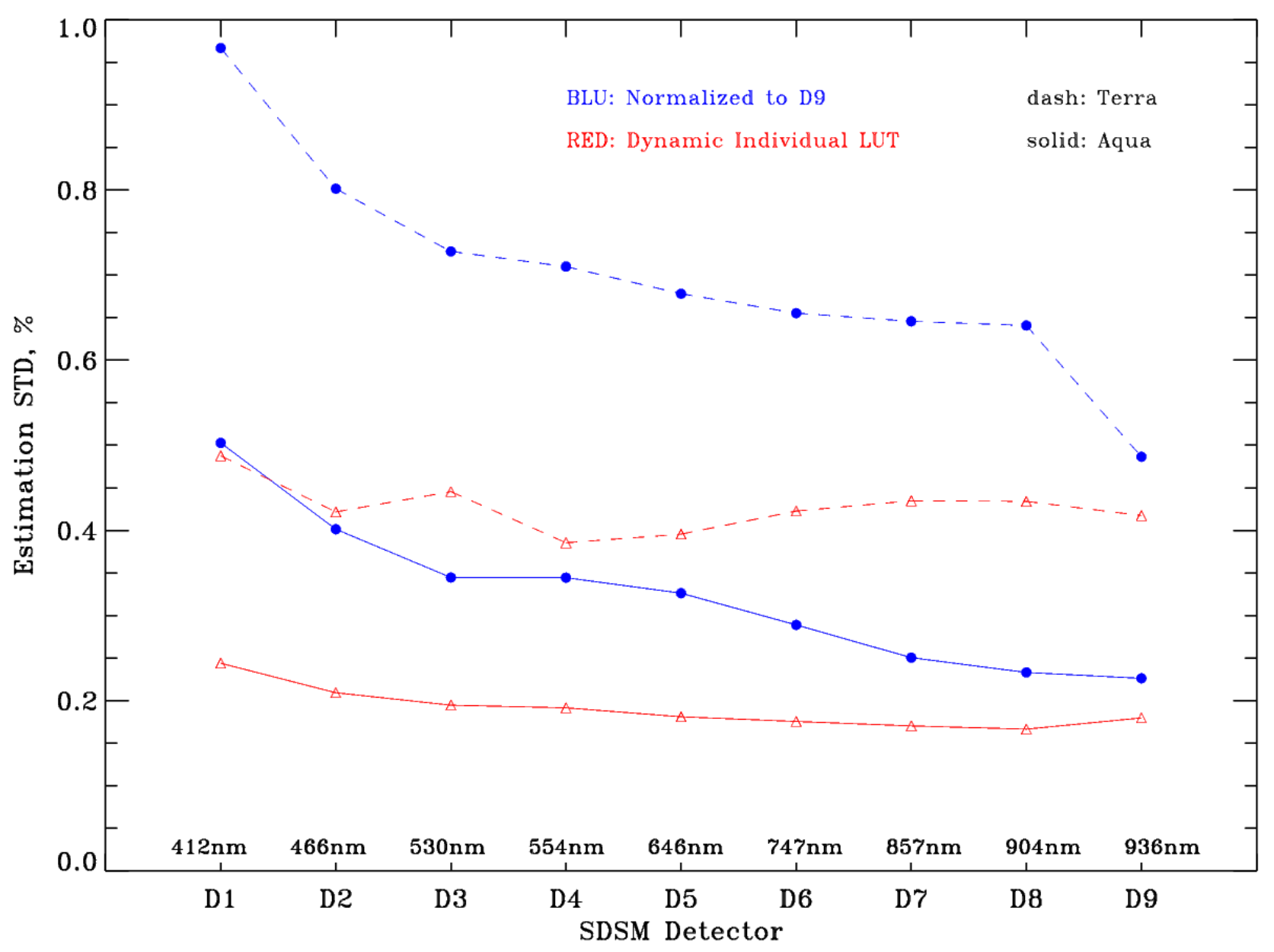

Figure 12 Terra and Aqua SDSM estimation standard deviation in two different LUT-based methods. 

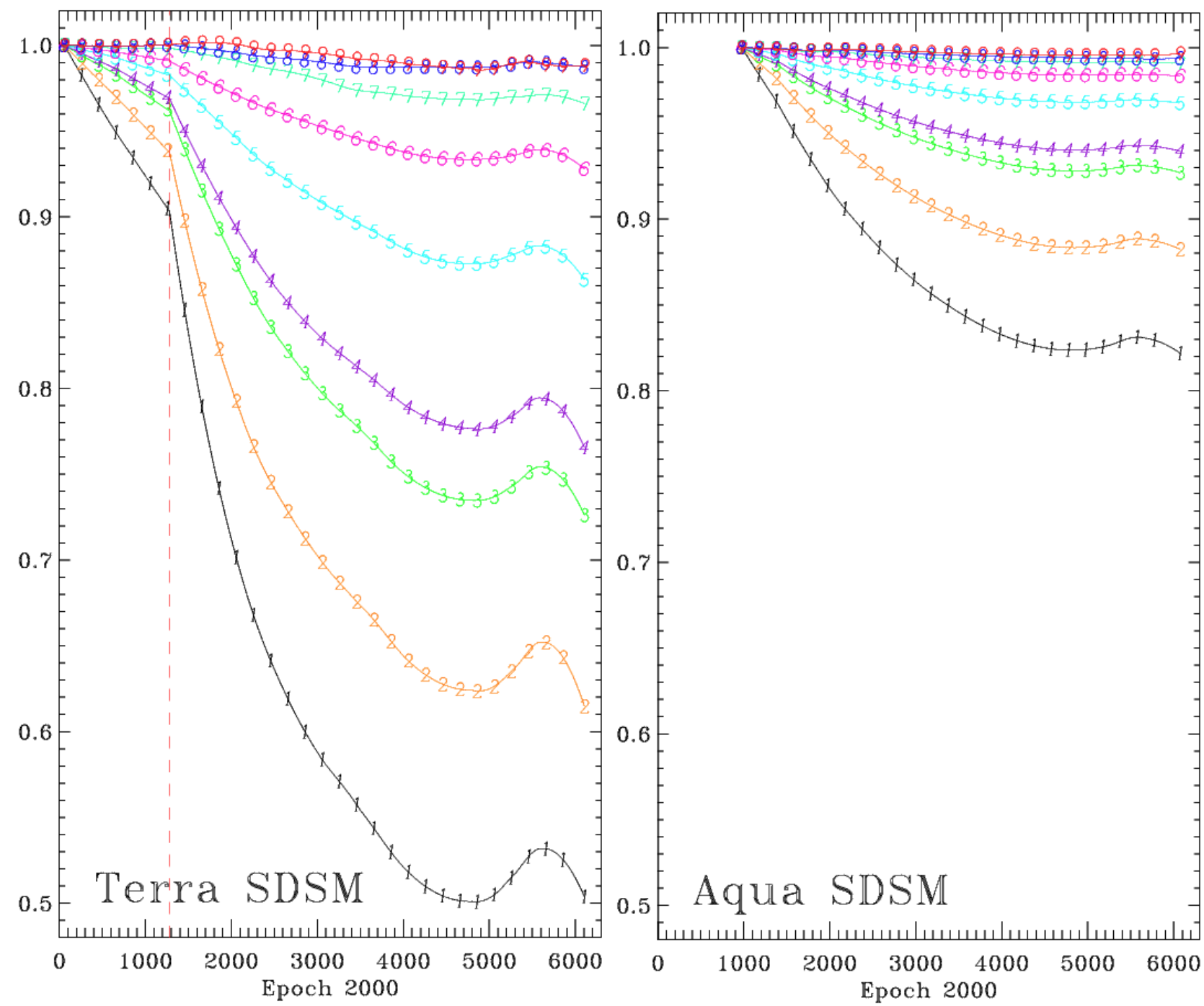

Figure 13 MODIS Terra and Aqua SDSM/SD degradation trending. 

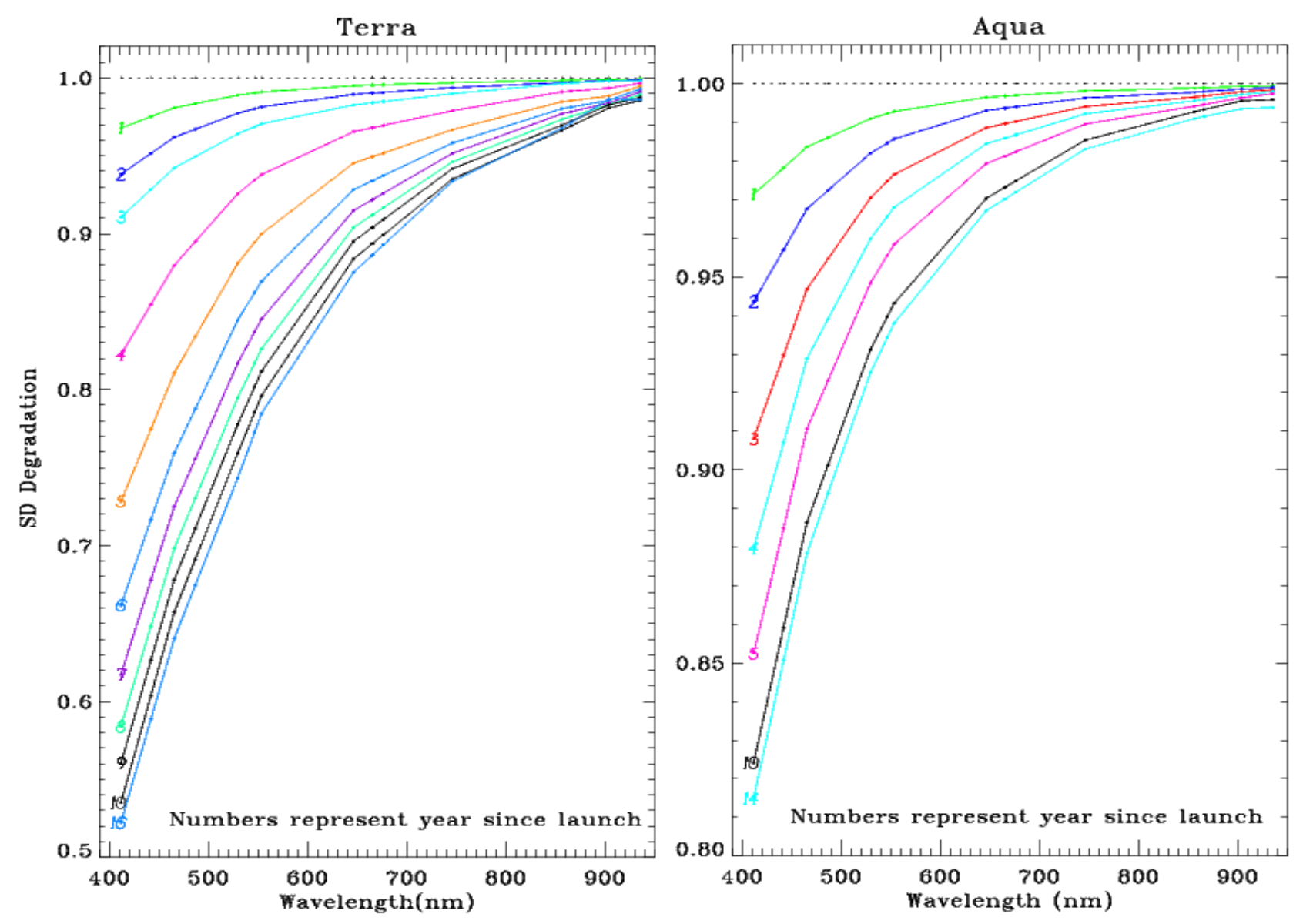

Figure 14 MODIS SD degradation. Colored curves indicate years since launch. 